QUADROS, R.M. et al. Ancylostoma spp. em cães de rua de Lages, Santa Catarina: variáveis epidemiológicas e coinfecção parasitária. PUBVET, Londrina, V. 8, N. 19, Ed. 268, Art. 1789, Outubro, 2014.

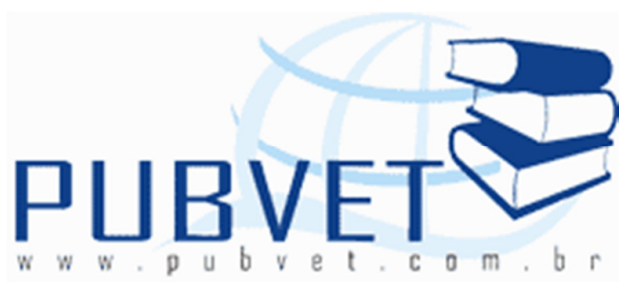

PUBVET, Publicações em Medicina Veterinária e Zootecnia.

\title{
Ancylostoma spp. em cães de rua de Lages, Santa Catarina: variáveis epidemiológicas e coinfecção parasitária
}

\section{Rosiléia Marinho de Quadros ${ }^{1}$, Fernanda Ronconi ${ }^{2}$, Sandra Marcia Tietz Marques $^{3 *}$, Paulo Henrique Exterchoter Weiss ${ }^{4}$, Marcio da Silva Orides ${ }^{5}$}

1 Médica Veterinária e Bióloga, Profa., Dra., Centro de Ciências Agroveterinárias da Universidade do Estado de Santa Catarina (CAV/UDESC)

${ }^{2}$ Bióloga - Universidade do Planalto Catarinense (UNIPLAC)

3 Médica Veterinária, PhD, Pesquisadora - FAVET/UFRGS. ${ }^{*}$ E-mail para correspondência: smtmuni@hotmail.com

${ }^{4}$ Biomédico - Mestrando - CAV/UDESC

${ }^{5}$ Médico Veterinário - Mestrando - CAV/UDESC

\section{Resumo}

A ancilostomose animal cursa com afecção gastrintestinal e respiratória, emagrecimento e retardo no desenvolvimento, podendo evoluir para caquexia e morte. Esta investigação avalia 357 amostras fecais de cães errantes apreendidos pelo Centro de Controle de Zoonoses (CCZ) do município de Lages, Santa Catarina, no período de junho de 2011 a janeiro de 2012, pelo método de Willis-Mollay. A prevalência de cães positivos para ovos de Ancylostoma spp. foi de 64,43\% (230/357) com 63,16\% (120/190) e 65,87\% (110/167), respectivamente, para fêmeas e machos. Animais com idades entre dois a cinco anos apresentaram maior infecção parasitária $(77,78 \%)$, porém 
QUADROS, R.M. et al. Ancylostoma spp. em cães de rua de Lages, Santa Catarina: variáveis epidemiológicas e coinfecção parasitária. PUBVET, Londrina, V. 8, N. 19, Ed. 268, Art. 1789, Outubro, 2014.

não houve diferença estatística entre as faixas etárias e sexo dos cães parasitados. A co-infecção parasitária entre Ancylostoma spp. foi observada com Trichuris vulpis e com Toxocara spp. O verão foi à estação que apresentou uma correlação significativa para a taxa de infecção por Ancylostoma spp. Dos 68 bairros da cidade, $44(64,71 \%)$ apresentaram cães infectados com Ancylostoma spp. Conclui-se que os cães errantes parasitados representam importante fonte de infecção, pois transitam pelas ruas e em praças públicas, sendo fundamental a adoção de medidas zoosanitárias, sobretudo na periferia da cidade, onde concentram-se em maior número.

Palavras-chaves: Ancylostoma, cães errantes, zoonose, epidemiologia

\section{Ancylostoma spp. in stray dogs of Lages, Santa Catarina, Brazil: epidemiological variables and parasite coinfection}

\section{Abstract}

Animal ancylostomiasis co-occurs with gastrointestinal and respiratory disorders, emaciation, and developmental delay, and may progress to cachexia and death. A total of 357 fecal samples from stray dogs captured by the Center for Zoonotic Disease Control in Lages, Santa Catarina, Brazil, from June 2011 to January 2012, were assessed by the Willis-Mollay method. The prevalence of positive dogs for Ancylostoma spp. eggs was 64.43\% (230/357), affecting $63.16 \%(120 / 190)$ of females and $65.87 \%(110 / 167)$ of males. Animals aged 2 to 5 years showed higher parasite infection rates $(77.78 \%)$, but no statistical difference was noted between age groups and sex of infected dogs. There was coinfection of Ancylostoma spp. with Trichuris vulpis and Toxocara spp. Summertime revealed a larger significant correlation with Ancylostoma spp. infection. Dogs infected by Ancylostoma spp. were detected in $44(64.71 \%)$ out of 68 neighborhoods. Infected stray dogs are an important source of infection as they roam the streets and public squares. Zoonotic control measures are therefore crucial, mainly on the outskirts, where the number of infected dogs is larger.

Keywords: Ancylostoma, stray dogs, zoonotic disease, epidemiology 
QUADROS, R.M. et al. Ancylostoma spp. em cães de rua de Lages, Santa Catarina: variáveis epidemiológicas e coinfecção parasitária. PUBVET, Londrina, V. 8, N. 19, Ed. 268, Art. 1789, Outubro, 2014.

\section{Introdução}

O papel do cão como hospedeiro definitivo de várias parasitoses de potencial zoonótico tem sido reconhecido de importância na saúde pública. Do ponto de vista epidemiológico, os cães errantes têm um papel importante na contaminação do meio ambiente, pois o fato de não receberem tratamento antiparasitário e pela livre circulação em áreas públicas, favorece a disseminação de formas parasitárias. O risco de infecção principalmente para crianças tem aumentado com os anos, sobretudo com o crescente número de cães domiciliados, peridomiciliados e errantes que vagueiam nos locais de lazer (GUIMARÃES et al., 2005).

Entre os principais parasitos causadores de zoonoses, encontrados em locais de lazer como praças e parques públicos está a ancilostomose, cujos excrementos de cães contendo os ovos do nematódeo podem infectar humanos causando a Larva Migrans Cutânea (LMC), também conhecida por dermatide serpiginosa ou bicho geográfico. O potencial zoonótico decorrente da LMC é maior para crianças que brincam em contato direto com solo, como as praias e caixas de areia de parques de recreação (SANTARÉM et al., 2004), sobretudo em solo arenoso que proporciona excelente microclima para as larvas, uma vez que elas ficam protegidas da radiação solar (NUNES et al., 2000).

Pela carência de dados sobre a ancilostomose na cidade de Lages, Santa Catarina, sul do Brasil, este trabalho objetivou conhecer a ocorrência de ovos de Ancylostoma spp. em cães errantes na qual podem ser um importante disseminador do parasito para humanos, sobretudo crianças que brincam em áreas públicas na cidade.

\section{Material e métodos}

Foram coletadas fezes de cães recolhidos pelo CCZ entre o mês de junho de 2011 a janeiro de 2012. O trabalho foi aprovado pelo comitê de ética em experimentação animal do Centro de Ciências Agroveterinárias da Universidade do Estado de Santa Catarina (UDESC), sob protocolo 134/2011. O CCZ é uma 
QUADROS, R.M. et al. Ancylostoma spp. em cães de rua de Lages, Santa Catarina: variáveis epidemiológicas e coinfecção parasitária. PUBVET, Londrina, V. 8, N. 19, Ed. 268, Art. 1789, Outubro, 2014.

instituição pública mantida pela Prefeitura Municipal da cidade de Lages, pertencente à Secretaria Municipal da Saúde, responsável pela prevenção e controle de zoonoses no município, desenvolvendo sistemas de vigilância sanitária e epidemiológica.

Amostras fecais foram coletadas diretamente do reto de 357 cães apreendidos pelo CCZ e encaminhadas ao Laboratório de Parasitologia da Universidade do Planalto Catarinense (UNIPLAC), processadas num período máximo de 24 horas. Os exames fecais foram realizados utilizando-se o método de flutuação simples através da técnica de Willis-Mollay, que utiliza solução saturada de cloreto de sódio (densidade $=1.182$ ) e a visualização dos ovos foi em microscopia óptica com objetiva de 10X.

A determinação da faixa etária estimada dos animais foi realizada pela análise da arcada dentária, considerando animais jovens até 12 meses de idade, adultos entre um ano e sete anos e idosos acima desta faixa etária (BRESCIANI et al., 2008).

A contagem de ovos nas amostras fecais foi realizada de acordo com a descrição de Hoffmann (1987), assim estabelecida: de 1 - 3 ovos (raríssimos); 3 - 5 ovos (raros); 6 - 10 ovos (pequena quantidade); 11 - 20 (regular quantidade); 21 - 50 ovos (grande quantidade) e acima de 51 indica intensa quantidade de ovos. Para este trabalho consideramos o grau de infecção parasitária através da contagem de ovos de 1 - 5 ovos por lâmina (+), 6 - 10 ovos $(++), 11-20$ ovos $(+++)$ e acima de 20 ovos $(++++)$. Também foi observada a consistência fecal, classificando entre normal e firme, com sinal de diarreia ou disenteria.

Segundo as informações de 2012 da Secretaria de Planejamento (SEPLAN) da Prefeitura Municipal de Lages, a cidade conta com 68 bairros. Para analisar os dados dos diferentes bairros, a cidade foi dividida em regiões para o melhor entendimento dos resultados: 1- região central; 2- região centro-oeste; 3 - região nordeste; 4 - região norte; 5 - região oeste; 6 região sudeste e 7 - região sul. 
QUADROS, R.M. et al. Ancylostoma spp. em cães de rua de Lages, Santa Catarina: variáveis epidemiológicas e coinfecção parasitária. PUBVET, Londrina, V. 8, N. 19, Ed. 268, Art. 1789, Outubro, 2014.

Para a análise estatística foi usado o teste $X^{2}$ pelo programa Software $R$ ( $R$ Core Team, 2012), adotando $p \leq 0,05$ como nível de significância com intervalo de confiança de $95 \%$ (IC).

\section{Resultados}

Das 357 amostras fecais de cães de rua apreendidos pelo Centro de Controle de Zoonoses (CCZ) de Lages, 230 (64,435\%) continham ovos de Ancylostoma spp. Das 357 amostras fecais analisadas nenhuma delas apresentou sinal de diarreia ou disenteria. Em relação ao sexo dos animais 190 eram de fêmeas e 167 de machos, das quais a positividade em relação ao sexo e faixa etária estão apresentadas na Tabela 1.

Tabela 1. Idades, sexo e positividade para Ancylostoma spp. dos cães apreendidos pelo CCZ na cidade de Lages, SC, entre o mês de junho de 2011 a janeiro de 2012.

\begin{tabular}{ccccc}
\hline $\begin{array}{c}\text { Classe } \\
\text { etária }\end{array}$ & \multicolumn{2}{c}{ Fêmeas } & \multicolumn{2}{c}{ Machos } \\
\cline { 2 - 5 } (anos) & $\mathbf{N}$ & $\begin{array}{c}\text { Positivos } \\
(\%)\end{array}$ & $\mathbf{N}$ & $\begin{array}{c}\text { Positivos } \\
(\%)\end{array}$ \\
\hline $0-1$ & 38 & $24(63,16)$ & 25 & $11(44)$ \\
$2-5$ & 122 & $76(62,30)$ & 92 & $67(72,83)$ \\
$6-8$ & 14 & $11(78,57)$ & 31 & $23(74,19)$ \\
$9-10$ & 00 & 00 & 12 & $06(50)$ \\
$>10$ & 06 & $03(50)$ & 06 & $03(50)$ \\
NI & 10 & $06(60 \%)$ & 01 & $00(100)$ \\
\hline & & $\mathbf{1 2 0}$ & & $\mathbf{1 1 0}$ \\
TOTAL & $\mathbf{1 9 0}$ & $\mathbf{( 6 3 , 1 6 \% )}$ & $\mathbf{1 6 7}$ & $\mathbf{( 6 5 , 8 7 )}$ \\
\hline
\end{tabular}

Nas categorias extremas de grau de infecção registrou-se o maior número de amostras, sendo que em $42,17 \%$ (97) das amostras o grau de 
QUADROS, R.M. et al. Ancylostoma spp. em cães de rua de Lages, Santa Catarina: variáveis epidemiológicas e coinfecção parasitária. PUBVET, Londrina, V. 8, N. 19, Ed. 268, Art. 1789, Outubro, 2014.

infecção foi baixo e em $27,83 \%$ (64) a infecção foi severa, conforme mostra a Figura 1.

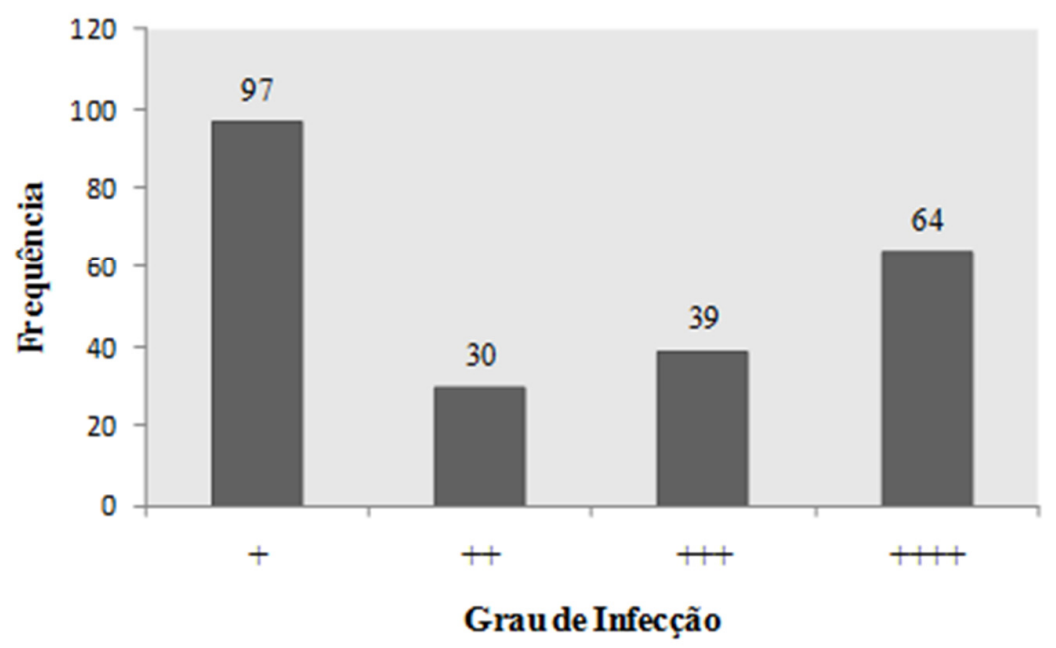

Figura 1. Número de amostras de acordo com o grau de infeç̧ão, em cães apreendidos pelo CCZ de Lages, SC, entre o mês de junho de 2011 a janeiro de 2012.

A ocorrência de Ancylostoma spp. em relação à estação do ano foi registrada conforme mostra a Tabela 2 .

Tabela 2. Prevalência de Ancylostoma spp. em relação a grau de infecção nas amostras fecais de cães apreendidos pelo CCZ de Lages, SC, em relação às estações do ano.

\begin{tabular}{lcccc}
\hline $\begin{array}{l}\text { Estação do } \\
\text { Ano }\end{array}$ & Grau 1 & Grau 2 & Grau 3 & Grau 4 \\
\hline Inverno & 22 & 05 & 06 & 18 \\
Primavera & 21 & 06 & 05 & 04 \\
Verão & 55 & 16 & 25 & 30 \\
Outono & 03 & 03 & 03 & 12 \\
\hline TOTAL & $\mathbf{9 7}$ & $\mathbf{3 0}$ & $\mathbf{3 9}$ & $\mathbf{6 4}$ \\
\hline
\end{tabular}

Entre os 68 bairros da cidade de Lages, $44(64,71 \%)$ apresentaram cães infectados com Ancylostoma spp. (Figura 2), porém não houve diferença estatística em relação à procedência dos animais. 
QUADROS, R.M. et al. Ancylostoma spp. em cães de rua de Lages, Santa Catarina: variáveis epidemiológicas e coinfecção parasitária. PUBVET, Londrina, V. 8, N. 19, Ed. 268, Art. 1789, Outubro, 2014.

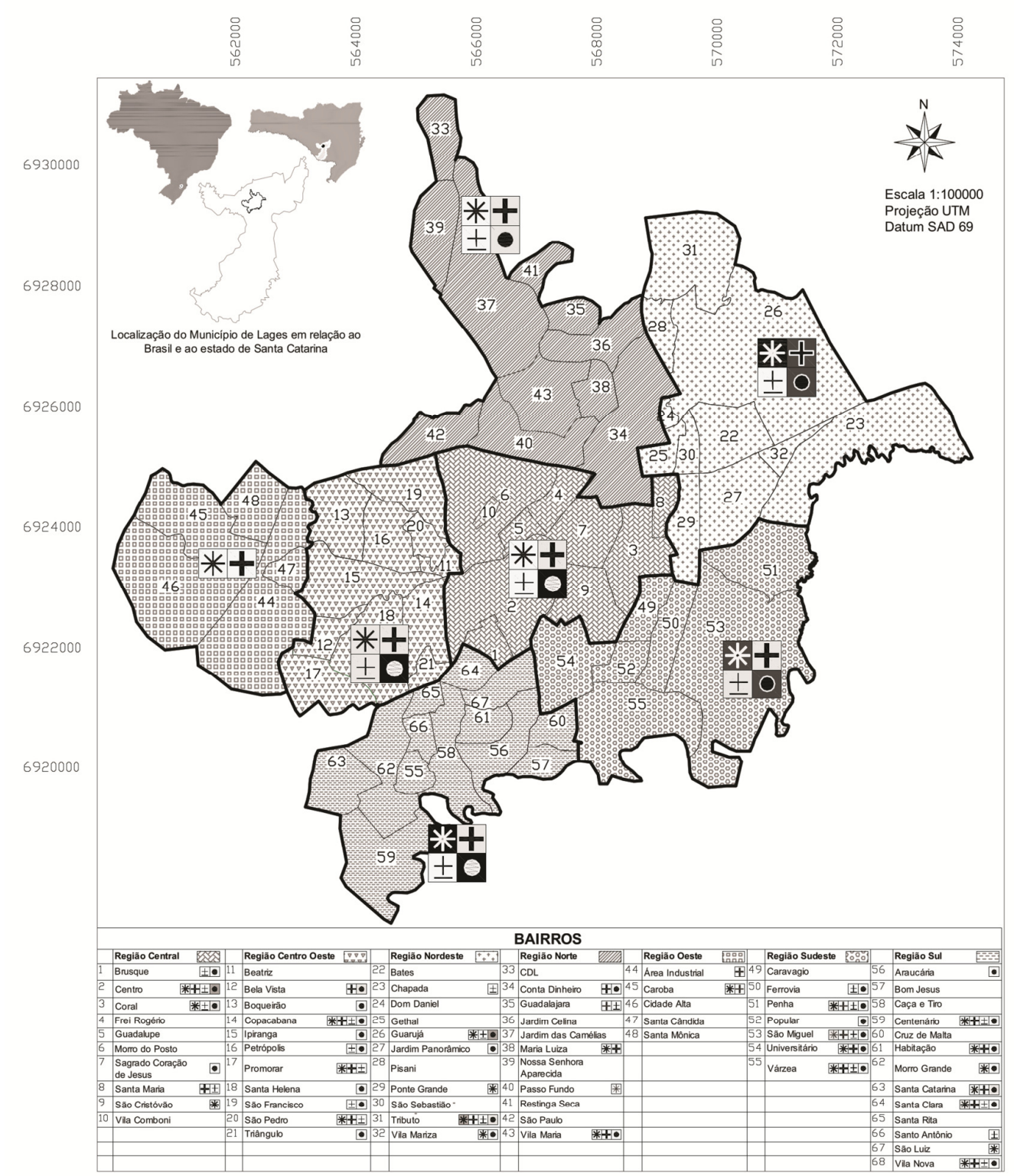

Figura 2. Bairros de procedência dos cães apreendidos pelo CCZ de Lages, SC e o grau de infecção parasitária.

\section{Discussão}

O diagnóstico parasitológico de ovos de Ancylostoma spp. pode apresentar diferentes prevalências que estão relacionadas com variáveis como as condições ambientais, temperatura, umidade e tipo de solo, fundamentais para a distribuição do parasito. A oscilação da presença do nematódeo ocorre, 
QUADROS, R.M. et al. Ancylostoma spp. em cães de rua de Lages, Santa Catarina: variáveis epidemiológicas e coinfecção parasitária. PUBVET, Londrina, V. 8, N. 19, Ed. 268, Art. 1789, Outubro, 2014.

sobretudo da procedência das amostras, como animais errantes ou domiciliados ou ainda se as fezes são coletadas do solo de praças públicas ou areia de praias (MCCARTHY; MORRE, 2000).

No Brasil, destacam-se trabalhos de avaliação dos aspectos epidemiológicos nos estados da federação com distintas prevalências, como 45,9\% (CASTRO et al., 2005), 41,7\% (CAPUANO e ROCHA, 2006), 53,1\% (TÁPARO et al., 2006), 75,38\% (BRESCIANI et al., 2008), 87,8\% (COELHO et al., 2011) no Estado de São Paulo; 71,3\% (SCAINI et al., 2003) no Rio Grande do Sul; $29,17 \%$ (LEITE et al., 2004) e 18,5\% (PRATES et al., 2009) no Paraná; 70,9\% (BLAZIUS et al., 2005), 37,93\% (BLAZIUS et al., 2006) e 17\% (PEDRASSANI et al., 2008) em Santa Catarina; 22\% (ALVES et al., 2005) e 45,3\% (OLIVEIRA et al., 2009) em Goiás e por Milken et al. (2007) na cidade de Uberlândia (MG) identificaram ovos do parasito em $17,46 \%$ em cães com idades até 12 meses e 11,11\% em animais com idades acima desta idade.

O elevado número de ovos presentes nas fezes analisadas, com a maioria apresentando mais de 20 ovos na lâmina foi proveniente de fêmeas $(31,66 \%)$ e cães na faixa etária entre dois a cinco anos de idade foram os que apresentaram maior número de ovos de Ancylostoma spp. (30,01\%), entretanto não foi possível justificar, pois não foram complementados com exames de sangue, embora as prevalências fossem muito semelhantes entre as taxas de infecção. Blazius et al. (2005) citam que todas faixas etárias podem estar infectados, pois não desenvolvem imunidade contra o nematódeo.

A alta prevalência de ovos de Ancylostoma spp. apresentada neste estudo pode ser em decorrência da procedência destes animais, com 95,24\% (340/357) provenientes de cães de bairros periféricos da cidade e que não recebem tratamento com anti-helmínticos. Além disso, os cães apresentavam deficiência nutricional o que pode ser explicado pela presença da alta infecção parasitária em todos as faixas etárias, não havendo diferença estatística, também observado em relação a cães machos e fêmeas, concordando com o resultado de Milken et al. (2007) com cães provenientes de canis em Uberlândia (MG). 
QUADROS, R.M. et al. Ancylostoma spp. em cães de rua de Lages, Santa Catarina: variáveis epidemiológicas e coinfecção parasitária. PUBVET, Londrina, V. 8, N. 19, Ed. 268, Art. 1789, Outubro, 2014.

Das 357 amostras fecais analisadas nenhuma delas apresentou sinal de diarreia ou disenteria, podendo ser atribuída a maior resistência do hospedeiro à infecção parasitária, traduzido pela capacidade de o animal conviver com Ancylostoma spp., onde cães de rua podem apresentar maior resistência e maior capacidade de hospedar o parasito em seu organismo, sem apresentar sinais clínicos (OLIVEIRA et al., 2009).

A infecção parasitária de Ancylostoma spp. com Trichuris vulpis foi observada em 6,22\% (35) dos cães errantes e de 2,22\% (15) com Toxocara spp. e o poliparasitismo foi diagnosticado em 5,22\% (12) com a associação entre Ancylostoma spp., Trichuris vulpis e Toxocara spp. e entre Ancylostoma spp., Trichuris vulpis e Cystoisospora spp. Estas associações também foram observadas por Scaini et al. (2003), Alves et al. (2005), Brener et al. (2005) e Milken et al. (2007).

No verão foi observado que os cães apresentaram maior grau de infecção pelo Ancylostoma spp. revelando uma diferença significativa pelo teste $x^{2}$ com $p=0,0004162$ em relação as demais estações. Este resultado pode ser meramente ao acaso, uma vez que podem não retratar o verdadeiro risco de infecção; porém para Naumova et al. (2007), alterações ecológicas como mudanças climáticas podem influenciar o aparecimento e proliferação de doenças parasitárias.

Com relação à patogenicidade, Bentubo et al. (2006) relacionaram as enfermidades parasitárias responsáveis por $41 \%$ das mortes de cães no município de São Paulo. Robertson et al. (2000) relataram que em países desenvolvidos as pesquisas parasitológicas de prevalências em cães e gatos portadores de helmintos gastrintestinais mostraram queda ao longo dos anos, cujo decréscimo pode estar associado à administração de medicamentos mais eficazes e seguros, bem como medidas diagnósticas mais confiáveis. Do ponto de vista epidemiológico, a redução das infecções parasitárias depende também de programas educacionais, mudanças comportamentais, ambientais e ecológicas a serem implementadas para reduzir estes índices e por consequência, o risco de sua transmissão. Para controlar os parasitos 
QUADROS, R.M. et al. Ancylostoma spp. em cães de rua de Lages, Santa Catarina: variáveis epidemiológicas e coinfecção parasitária. PUBVET, Londrina, V. 8, N. 19, Ed. 268, Art. 1789, Outubro, 2014.

zoonóticos, sobretudo na redução da presença de seus ovos no ambiente são necessários recursos financeiros adequados que precisam ser alocados para manter os programas que reduzam a contaminação (ROBERTSON et al., 2000).

O conhecimento da ocorrência do parasitismo com características zoonóticas em animais domésticos, principalmente os que transitam livremente em áreas urbanas, são de suma importância principalmente para crianças que são consideradas grupo de risco, já que têm contato mais frequente com solo de parques e áreas públicas (MARTINEZ-BARBABOSA et al., 2003).

\section{Conclusão}

O resultado mostra alta prevalência do parasito, elevada taxa de infecção e a falta de sinal clínico evidente. Conclui-se que os cães apreendidos pelo CCZ apresentam grande potencial para transmitir Ancylostoma spp. e outros parasitos gastrintestinais a outros animais ou mesmo humanos, visto que estes animais transitam pelas ruas, sobretudo em praças públicas, sendo importante medidas zoosanitárias para eliminar as parasitoses intestinais sobretudo na periferia da cidade, onde estes cães encontram-se em maior número e sem assistência sanitária.

\section{Referências}

Alves, O. F.; Gomes, A. G.; Silva, A. C. 2005. Ocorrência de enteroparasitos em cães do município de Goiânia, Goiás: Comparação de técnicas de diagnóstico. Ciência Animal Brasileira, 6(2): 127-133.

Bentubo, H. D.,L.; Tomaz, M. A.; Bondan, E. F.; Lallo, M. A. 2007. Expectativa de vida e causas de morte em cães na área metropolitana de São Paulo (Brasil).Ciência Rural, 37(4): 1021-1026.

Blazius R.D., Emerick S., Prophiro J.S., Romão P.R.T. \& Silva O.S. 2005. Ocorrência de protozoários e helmintos em amostras de fezes de cães errantes da Cidade de Itapema, Santa Catarina. Rev. Soc. Bras. Med. Tropical. 38(1): 73-74.

Blazius R.D., Silva O.S., Kauling A.L., Rodrigues D.F.P. \& Lima M.C. 2006. Contaminação da areia do Balneário de Laguna, SC, por Ancylostoma spp., e Toxocara spp. em amostras fecais de cães e gatos. Arquivos Catarinenses de Medicina. 35(3): 55-58. 
Brener B., Lisboa L., Mattos D. P. B. G., Arashiro E. K. N., Millar P. R., Sudré A. P. \& Duque V. 2005. Frequência de enteroparasitas em amostras fecais de cães e gatos dos municípios do Rio de Janeiro e Niterói. $R$. bras. Ci. Vet. 12 (1/3): 102-105.

Bresciani K.D.S, Ishizaki M.N., Kaneto C.N., Montano T.R.P., Perri S.H.V., Vasconcelos R.O. \& Nascimento A.A.D.O. 2008. Frequência e intensidade parasitária de helmintos gastrintestinais em cães na área urbana do Município de Araçatuba, SP. ARS VETERINARIA. 24(3): 181-185.

Capuano D.M \& Rocha G.M. 2006. Ocorrência de parasitas com potencial zoonótico em fezes de cães coletadas em áreas públicas do município de Ribeirão Preto, SP, Brasil. Rev. Bras. Epidemiol. 9(1): 81- 86.

Castro J.M., Santos S.V \& Monteiro N.A. 2005. Contaminação da orla marítima do Município de Praia Grande, São Paulo, por ovos de Ancylostoma e Toxocara em fezes de cães. Rev. Soc. Bras. Med. Tropical. 38(2): 199-201.

Coelho W.M.D., Amarante A.F.T, Apolinário J.C., Coelho N.M.D. \& Bresciani K.D.S. 2011. Ocurrence of Ancylostoma in dogs, cats and public places from Andradina city, São Paulo, Brazil. Rev. Inst. Med. Trop. 53(4): 181-184.

Scaini C.J., Toledo R.N., Lovatel R., Dionello M.A., Gatti F.A. \& Susin L. 2003. Contaminação ambiental por ovos e larvas de helmintos em fezes de cães na área central do Balneário de Cassino, Rio Grande do Sul. Rev. Soc. Bras. Med. Tropical. 36(5): 617-619.

Chomel B. B. 2008. Control and prevention of emerging parasitic zoonoses. International Journal for Parasitology. 38 (11):1211-1217.

Guimarães A.M., Alves E.G.L., Rezende G.F. \& Rodrigues M. 2005. Ovos de Toxocara sp. e larvas de Ancylostoma sp. em praça pública de Lavras, MG. Rev. Saúde Pública. 39(2): 293295.

Hoffmann R.P. 1987. Diagnóstico de Parasitismo Veterinário. Porto Alegre: Sulina, 156p.

Leite L.C., Marinoni L.P., Cirio S.M., Diniz J.M.F., Silva M.A.N., Luiz E., Molinari H.P., Vargas C.S.G., Leite S.C., Zadorosnei, A.C.B \& Veronesi E.M. 2004. Endoparasitas em cães (Canis familiaris) na cidade de Curitiba-Paraná-Brasil. Archives of Veterinary Science. 9(2): 95-99.

Martinez-Barbabosa I., Vázquez Tsuji O., Cabello R.R., Cárdenas E.M. \& Chasin O.A. 2003. The prevalence of Toxocara cati in domestic cats em Mexico City. Veterinary Parasitology. 114(1): 43-49.

McCarthy J. \& Morre T.A. 2000. Emerging helminth zoonoses. Int. J. Parasitol. 30(12-13): 1351-1360.

Milken V.M.F., Cunha G.N., Cabral A.D \& Cabral D.D. 2007. Endoparasitoses em canis do munícipio de Uberlândia, Minas Gerais. Acta Scientiae Veterinariae. 35(Supl.2): 707-708.

Naumova E.N., Jagai J.S., Matyas B., Demaria J.R., Macneill I. B. \& Griffiths J.K. 2007. Seasonality in six enterically transmitted diseases and ambient temperature. Epidemiol. Infect. 135(2): 281-292.

Nunes C. M. , Pena F.C., Negrelli, G.B., Anjo C.G.S., Nakano M.M. \& Stobbe N.S. 2000. Ocorrência de larva migrans na areia de áreas de lazer das escolas municipais de ensino infantil, Araçatuba, SP, Brasil. Revista de Saúde Pública. 34(6): 656-658. 
Oliveira V.S.F., Melo D.P.G., Fernandes P.R., Schulze C.M.B., Guimarães M.S. \& Silva A.C. 2009. Ocorrência de Helmintos gastrintestinais em cães errantes na cidade de Goiânia - Goiás. Rev. Patologia Tropical. 38 (4): 279-283.

Pedrassani D., Vieira A.M. \& Thiem E.M.B. 2008. Contaminação por Toxocara spp. e Ancylostoma spp. em área de lazer do município de Canoinhas, SC. Archives of Veterinary Sciences.13(2): 110-117.

Prates L., Pacheco L.S., Kuhl J.B., Dias M.L.G.G., Araújo S.M. \& Pupulin A.R.T. 2009. Frequência de parasitos intestinais em cães domiciliados da cidade de Maringá, PR. Arq. Bras. Med.Vet. Zootec. 61(6): 1468-1470

R Core Team. 2012. R: Language and environment for statistical computing. R. Foundation Statistical Computing, Viena, Áustria. ISBN 3- 900051-07-0, URL Disponível em $<$ http://www.R-project.org/

Robertson I. D., Irwin P. J., Limbery A. J. \& THOMPSON R. C. A. 2000. The role of companion animals in the emergence of parasitic zoonoses. International Journal for Parasitology. 30(1213): $1369-1377$.

Santarém V.A., Giuffrida R. \& Zanin G.A. 2004. Larva Migrans Cutânea: ocorrência de caos humanos e identificação de larvas de Ancylostoma spp. em parque público do município de Taciba, São Paulo. Rev. Soc. Bras. Med. Tropical. 37(2): 179-181.

Táparo C.V, Perri S.H.V., Serrano A.C.M., Ishizaki M.N., Costa T.P, Amarante A.F.T. \& Bresciani K.D.S. 2006. Comparação entre técnicas coproparasitológicas no diagnóstico de ovos de helmintos e oocistos de protozoários em cães. Rev. Bras. Parasitol. Vet. 15(1): 1-5. 